\title{
Influenza: An Emerging and Re-emerging Public Health Threat in Nepal
}

\author{
Upadhyay Bishnu Prasad ${ }^{1,2}$
}

\author{
Affiliations: \\ ${ }^{1}$ National Public Health Laboratory, Government of \\ Nepal \\ ${ }^{2}$ Central Diagnostic Laboratory \& Research Center, Pvt. \\ Ltd, Kathmandu Nepal
}

\section{Correspondence to:}

Bishnu Prasad Upadhyay

Microbiologist, In-Charge: NIC, Molecular Diagnostics \& BSL-3 Laboratory

National Public Health Laboratory, Govt. of Nepal. Chief Consultant Molecular Biologist

Central Diagnostic Laboratory \& Research Center

Kathmandu, Nepal

Email:bishnupd@gmail.com

\section{How to cite this Article:}

Influenza: An Emerging and Re-emerging Public Health Threat in Nepal. Bishnu Prasad Upadhyay. Ann. Clin. Chem. Lab. Med. 2017:3(2);1-2

DOI: http://dx.doi.org/10.3126/acclm.v3i2.20737

\section{(C) 2017 Nepalese Association for Clinical Chemistry}

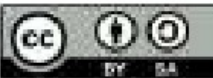

This work is licensed under a Creative Commons Attribution-Share Alike 4.0 International License.
Influenza virus type $\mathrm{A}$ and $\mathrm{B}$ are responsible for seasonal epidemics as well as pandemics in human ${ }^{1}$. Influenza A viruses are further divided into two major groups namely, low pathogenic seasonal influenza (A/H1N1, A/H1N1 pdm09, A/H3N2) and highly pathogenic influenza virus (H5N1, H5N6, H7N9) on the basis of two surface antigens: hemagglutinin (HA) and neuraminidase (NA). Mutations, including substitutions, deletions, and insertions, are one of the most important mechanisms for producing new variant of influenza viruses ${ }^{2}$. During the last 30 years; more than 50 viral threat has been evolved in South-East Asian countries ${ }^{3,4,5}$ of them influenza is one of the major emerging and re-emerging infectious diseases of global concern. Similar to tropical and sub-tropical countries of Southeast Asia; circulation of $\mathrm{A} / \mathrm{H} 1 \mathrm{~N} 1$ pdm09, A/H3N2 and influenza B has been circulating throughout the year with the peak during July-November in $\mathrm{Nepal}^{6}$. However; the rate of infection transmission reach peak during the post-rain and winter season of Nepal.

Influenza transmission occurs predominately by coughing or sneezing. Transmission also occurs through direct contact with respiratory droplets/secretions or contaminated objects followed by touching the nose, mouth or eyes. More than 100 viruses are capable of causing respiratory infections with similar symptoms of fever, chills, running nose, sore throat, headache, tiredness, dry cough and some time breathing difficulties ${ }^{7}$. Clinically, it is very hard to differentiate and confirm the etiology for empirical therapy.

In healthy individuals; viral fever may persist up to one week and usually resolved by immune system without specific medication. However; it may lead to pneumonia and acute respiratory distressed syndrome (ARDS) in children under five years of age, pregnancy, immuno-compromised hosts, patients with chronic diseases and elderly peoples ${ }^{8,9}$.

Nasal/throat swab or nasopharyngeal specimen needs to be investigated within 2-3 days of symptoms for confirmatory diagnosis by real time PCR method. Such facility is confined at National Public Health Laboratory in Kathmandu. The facility need to be expanded throughout the country who are illegible to fulfill the required laboratory criteria at medical institute, regional hospitals, stand-alone research laboratory and public private sectors. 
At the time of specimen collection, packaging and shipment; gown, glove, mask and face shield must be worn. All health care provider working at outpatient/ inpatient department, Operation Theater and critical care units are requested to maintain proper hand hygiene and use of personal protective equipment. Hand hygiene is the most effective and important measure in preventing the spread of influenza. The use of clean soap-water and or alcohol-based hand rub is the most effective method of hand hygiene. The knowledge of influenza and its consequences should be incorporated in different level of academia and training programs in Nepal.

\section{REFERENCES}

1. Upadhyay BP, Ghimire P, Tashiro M and Banjara MR. Molecular epidemiology and antigenic characterization of seasonal influenza viruses circulating in Nepal. J Nepal Health Res Counc. 2017;15 (35):44-50.

2. Venkatesh D, Poen M, Bestebroer TM et al. Avian influenza viruses in wild birds: virus evolution in a multi-host ecosystem. J Virol. 2018.

3. Gonzalez JP. Arboviruses and related viruses as emerging pathogens in Southeast Asia: International conference of emerging reemerging communicable diseases and impending epidemics, Bangkok17-19 July, 1995. Factors in the emergence of Arbovirus Diseases. 1995.

4. Wang LF and Crameri G. Emerging zoonotic viral diseases. Rev Sci tech Off int Epiz. 2014;33(2):56981.

5. Dash AP, Bhatia R, Sunyoto T and Mourya DT. Emerging and re-emerging arboviral disease in Southeast Asia. J Vector Borne Dis. 2013:77-84.

6. Upadhyay BP, Ghimire P, Tashiro M and Banjara MR. Characterization of seasonal influenza virus types and sub-types isolated from influenza like illness cases of 2012. Kathmandu Univ Med J. 2017;57(1):56-60.

7. Rossetto EV and Luna EJ. A descriptive study of pandemic influenza A(H1N1) pdm09 in Brazil, 2009-2010. Rev Inst Med Trop Sao Paulo. 2016;58 (78).

8. Javid N Moradi A, Tabarraei A and Bazouri M. Clinical and epidemiological profile of pandemic influenza AH1N1, H3N2 and type B in the Southern of Caspian sea, Iran. Jundishapur J Microbiol. 2017.

9. Ma HY, Wu JL, Lu CY, Chen JM, Lee PI Chang LY and Huang LM. Risk factors associated with severe influenza virus infection in hospitalized children during the 2013 to 2014. J Microbiol Immunol Infect. 2016;49(3):387-93. 